\title{
The Role of Parametric Design in Enhancing the Blast Resistance of Geometries
}

\author{
Sarkawt Azad Noori
}

\begin{abstract}
This paper draws on the findings of an on-going doctoral research into the potential of parametric design in reducing the impact of blast wave on a number of shapes and geometries. Using Coyote tool, which interconnects the Grasshopper design environment with ANSYS Autodyn high-pressure impact simulator, firstly, the impact of blast wave on basic geometries such as cube is analyzed, and its main components (parameters) are addressed. Secondly, a series of parametrically design proposals are applied on the same geometry and then re-simulated. The findings show that when correctly considering the components of blast wave, parametric design can reduce the effect of blast on geometries. This paper suggests new design methods by which the impacts of blast and explosions on buildings can be mitigated.
\end{abstract}

Keywords - Parametric Design, blast wave pressure, blast load, simulation, Coyote tool.

\section{INTRODUCTION AND BACKGROUND}

The recent increase of terrorist attacks on important facilities in the heart of urban areas as a new type of war is constituting a great risk on both cities' residents and buildings, it is also implying more actions and studies in the field of urban design, architecture and civil engineering. There is a wide literature about design methods and guidelines of mitigating the danger of explosions and blasts on buildings and city in general, some of the studies are focusing on strengthening the buildings' structures against blast waves such as using modular structures [1], others on the behaviours of different shapes or forms of structural elements like: columns, beams ....etc against blast waves using finite element method simulation [2], and effective shapes against blast wave [3], also, there are manuals and guidelines for enhancing security and security management such as FEMA [4], or guides to design for security [5]. But, the complex nature of explosions and its multiple variables in addition to the limited access to real time experiments of explosions, especially for academic purposes, all of this requires seeking new methods or techniques of analyzing and experimenting. The recent advancements in computation software and simulation provide new virtual environments for experimenting, analyzing and visualizing such complex phenomenon. This on going study investigates the potentials of computer-aided design and specifically parametric design in mitigating the impact of explosion on building by testing the behaviour of parametrically designed shapes and forms against detonation and blast waves.

\footnotetext{
${ }^{1}$ Department of Civil and Environmental Engineering, The University of Auckland, New Zealand, Email: tfra054@aucklanduni.ac.nz
}

It is important here to mention that this paper focuses on explosions caused by bombs, which means those explosions that could be delivered by cars or can be placed near a targeted building as they are the common types of attacks in urban areas due to the relatively easiness of moving them around. Normally the amount of explosives, in this type, does not exceed $225 \mathrm{~kg}$ approximately [5]. This type of attacks could be foreseen, stopped or its impacts can be minimized by understanding the nature, behaviour of explosions and their propagation toward and around buildings, and accordingly determine the weak spots and vulnerability of design and structure [6].

\section{A. The Nature of Explosions}

An explosion produces air compression and resumption; When an explosive material is initiated a very rapid exothermic chemical reaction will occur, in the progress of this chemical reaction the solids and liquids contained in the explosive compound are converting to a very high dense gas, the product of this reaction expands at a very high velocity trying to reach an equilibrium state with the surrounding air, creating what is known as "shock wave" [4]. The shock wave, which is a high compressed air, travels at a super sonic speed radially from the centre of the detonation. Explosions, in general, can be visualized as spherical or hemispherical bubbles of gas, depending on whether the detonation is in the air or on ground respectively. As the expansion of the shock wave continues, pressures decrease exponentially over time, it is usually measured by milliseconds due to its short life-span, also pressures decreases over the cube of the distance because of the dissipation of energy and geometric divergence [4].

Abstractly explosions have two main phases; the positive and the negative phase, at the positive phase, explosions create an immediate rise of the ambient pressure toward peak overpressure also known as "incident pressure", as the shock wave expands pressure decreases towards ambient pressure. The negative phase starts once the pressure reaches ambient pressure, negative phase is usually longer and has less effect on its surrounding due to the little energy left form the ignition of the explosion. The two phases are shown in figure 1 . 


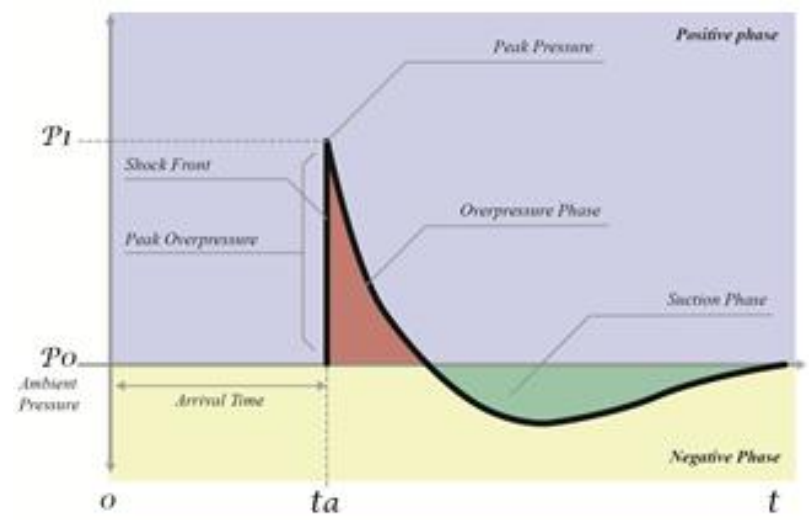

Fig. 1: Pressure time-history

Overpressure is the difference between the peak pressure $\mathrm{P} 1$ and the ambient pressure P0. When the blast wave propagates and hits a rigid object it cannot be reflected freely as the blast wave forces more air particles to follow, creating a phenomenon of dynamic pressure known as reflected overpressure. The maximum overpressure of the blast wave is called peak side-on overpressure $P s o$, and the maximum overpressure of the reflected blast wave is called peak reflected overpressure Pro, which is higher than the peak sideon overpressure [3,7]. The reflected overpressure is what is known as "design load" (in this case is blast load) in structural engineering, that has serious effect on buildings. Furthermore, distance and time play important role, the peak side-on overpressure decreases with distance from the centre of detonation [8]. The main parameters of blast load are peak overpressure and the duration of the overpressure phase also known as maximum impulse per unit area as in equation (1). The maximum impulse is the integral of force over time as in equation (2), thus in this case; it is the integral of overpressure time-history as in equation (3).

$$
\begin{aligned}
I_{\max } & =\max (I(t)) \\
I(t) & =\int F(t) d t \\
I(t) / A & =\int_{t_{a}}^{t_{a}+t} p(t)-p_{0}
\end{aligned}
$$

Approximating the overpressure model to two value of side-on overpressure or peak reflected overpressure and max impulse is not a complete representation of the blast load, but this simplification is easier for comparing and examining shapes and architectural models against blast load [3]. However, this approximation is not acceptable for examining flexible structures as the suction phase is ignored, also, it is not acceptable for internal explosions because of the multiple reflection occurrences and deflagration. In this paper only external explosions are focused on and therefore the approximation method is followed.

In this section only a general overview has been explained as it is a formidable task to cover all the information, equations and diagrams regarding blast behaviour. For further readings see for example M.Y.H. Bangash \& T. Bangash [7], G.F. Kinney and K.J. Graham [8], G.C. Mays and P.D. Smith [9].

\section{B. Reduction of blast load by form and shape}

Since this paper concentrates on the design of blast resistance based on form and shape, this section sheds the light on the related existing literature, albeit it is relatively limited. Every geometrical form reacts differently to blast wave, according to Bitarafian et al, [2] in their study on resistance of from by numeric element simulation; cylinder and cube are considered unsuitable for blast resistance, and hemisphere and pyramid forms provide a better resistance while conical form has the best resistance. Gebbeke and Döge [3] conducted an extensive study on the behaviour of different forms and shapes to blast load in both 2D and 3D using finite volume method showing that concave shapes can reduce the effect of blast load more effectively than convex shapes.

The angle between the direction shock wave and the reflecting surfaces, known as "angle of incidence", has a significant role in increasing or decreasing the reflected pressure; when the direction of blast wave is perpendicular to reflecting surfaces a maximum reflected pressure is experienced, however when the reflecting surface is parallel to the direction of the blast wave, the reflected pressure is minimum, usually angles between $40^{\circ}$ to $60^{\circ}$ experience an increase in reflected pressure [4], therefore it is important to consider the angle of incident in form and shape design.

\section{Parametric Design}

The advancement of computer science, over the last few decades, is influencing most of the fields and disciplines. New techniques and methods are trending in the field of architectural design known as computer aided design (CAD), digital design, parametric design, generative design and computational design, ...etc, some of which rely on computer as a drafting tool, while others use it for generating forms programmatically or algorithmically.

Woodbery [10] compares conventional design with parametric design, revealing the extra capabilities of the latter such as relating and repairing; design components relate to each other co-ordinately and when an indicator changes, all the other connected indicators change as well and they repair themselves accordingly, this way parametric design allows the designer to consider more variables while designing, and creating interrelations between them. These extra capabilities of parametric design enable it to undertake very complex operations that are not possible with the conventional analysis and design methods. This study hypothesizes the potential of parametric design in dealing with the multi-variables nature of explosions, and then designing accordingly.

\section{Coyote tool}

Coyote is an inter-process plug-in to intercommunicate between Grasshopper 3D - an algorithmic modelling add-on for Rhino 3D - as a design environment, and ANSYS Autodyn as a simulator for performing finite element volume simulation for modelling blast load on geometries. Coyote prepares the 3D models and all the other necessary information for performing the simulation including; explosive type, explosive weight, initial condition, boundary condition ....etc and sends them to Autodyn in order to start the simulation, after the simulation is done Coyote can read and visualize the results such as pressure throughout the domain, blast load on surfaces and pressure time-history inside Grasshopper 3D. In this way 
designers can test their models against blast wave and refine their designs. Coyote tool works as indirect tool [11], which facilitates the process of preparing the model for simulation and also retrieving the results from the simulation in order to pass them as parameters for design and perhaps conducting a refining process to achieve the expected design objectives.

\section{EXPERIMENTING AND ANALYSIS}

This section is composed of three parts; the first one is about introducing the geometrical model and data, the second one is about sending the data for initial simulation through the Coyote tool and retrieving the results which are: pressure throughout the domain and blast load, the third one is about redesigning the form parametrically based on the initial simulation results, and for this purpose three design options are proposed, then each design proposal is sent for simulation again through Coyote tool.

\section{A. Model and data}

For experimenting, a model has been prepared with a boundary domain of $45 \mathrm{~m} \times 36 \mathrm{~m} 26 \mathrm{~m}$, a cube form has been placed inside the domain with a volume of $4096 \mathrm{~m}^{3}$ and dimensions of $16 \mathrm{~m} \times 16 \mathrm{~m} \times 16 \mathrm{~m}$ (resembling a building), an explosive of $115 \mathrm{~kg}$ (average weight of bombs) of TNT has been placed at $15 \mathrm{~m}$ distance from the front face of the cube.

First, for measuring air pressure, each cube surface has been subdivided into $16 \times 16$ subdivisions, each of $1 \mathrm{~m}^{2}$ in order to match the subdivisions of the domain. Second, for measuring blast load on surfaces by using numerical analysis, each surface has been subdivided to $8 \times 8$ subdivisions, each of $2 \mathrm{~m}^{2}$ in order to distribute the gauges for reading blast load on the surfaces. Decreasing of cube subdivisions in this part is due to the limitation of Autodyn in having maximum number of 500 gauges per simulation.

\section{B. Initial simulation (the case of basic cube)}

After preparing the model with the characteristics mentioned in section (2.1), it was sent to Autodyn for simulation, the results have been read by Coyote tool for visualization as shown in figure 2 .

The left figure demonstrates the maximum pressure in timehistory throughout the domain and around the cube by visualizing the pressure of air next to each $1 \mathrm{~m}^{2}$ area on the surfaces; the highest pressure is $21000 \mathrm{MPa}$. The right figure shows the blast load reading in each gauge point on the surfaces of the cube.
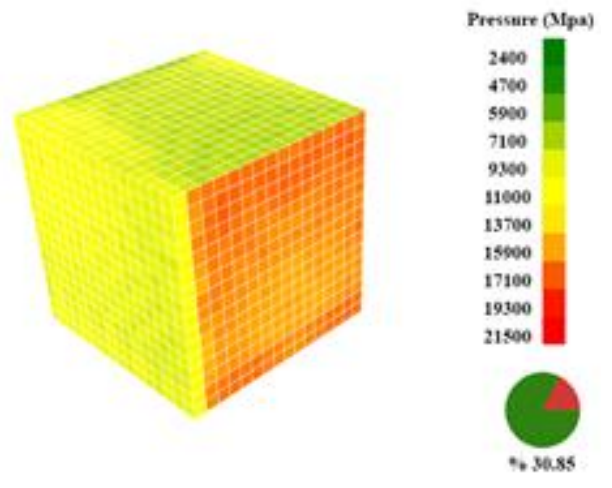
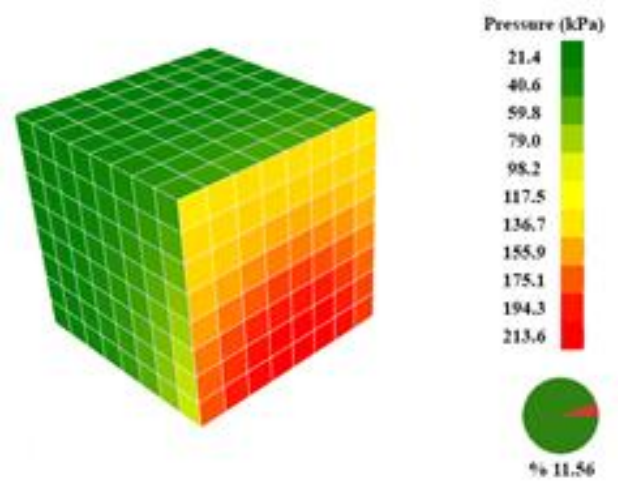

Fig. 2: Visualization of blast pressure throughout the domain and blast load on surfaces.

\section{Design proposals}

The distance from the explosive location, pressure around the form and blast load on surfaces have been considered as parameters, in addition to all the information mentioned in section (1.2). In this experiment pressure higher than $12600 \mathrm{MPa}$ is considered dangerous and worth redesigning (deciding the dangerous pressure usually depends on the materials and reinforcement systems adopted in buildings, for the purpose of this experiment - since no materials are considered- pressures higher than \%60 of the maximum pressure is assumed dangerous). Based on these parameters, three design proposals have been considered:

The first proposal, the surfaces of the cube are subdivided into subdivisions of $4 \mathrm{~m} \times 4 \mathrm{~m}$, then each surface with dangerous pressure has been taken under consideration to be

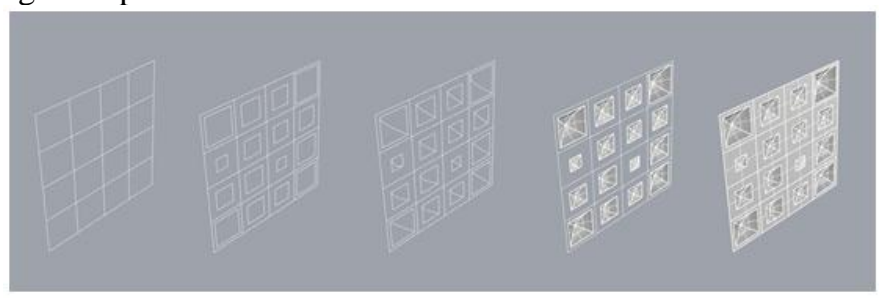

Fig. 3: First design proposal.

redesigned as shown in figure 3 , depending on the pressure by the surfaces, an offset operation has been applied in a way when the pressure is high the offset distance is lower (all pressures have been remapped), so less flat surfaces face the blast wave. Later the centre point of each new square generated from the offset operation has been pulled inward by the amount of the remapped value of pressure, and then an interpolated curve connects each two opposite corners passing through the pulled point, creating curves for forming concave surfaces.

For the second proposal, the subdivisions by dangerous pressure have been focused on, and then a diagonal curve between two corners of each subdivision has been selected and evaluated by the value of remapped pressure in order to determine a point on the diagonal curve, later these evaluated points have been moved outward according to the amount of applied pressure, finally a triangle mesh surface has been constructed by connecting each two neighbouring corners on each subdivision with the moved evaluated point, resulting in 
replacing each subdivision with a pyramid-like form as shown in figure 4.

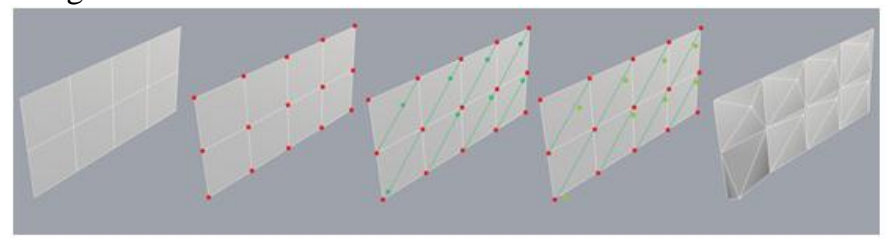

Fig. 4: Second design proposal

Regarding the third proposal, the pressure by each subdivision has been used as a parameter to determine an evaluation point on the vertical edges of each squared subdivision, then a line connecting the two points has been drown, using the remapped pressure value another evaluation point has been determined on the line, later the point has been pulled outward based on the amount of applied pressure, then an interpolated curve goes through evaluated point on one vertical edge to the opposite, passing through the pulled point, finally the upper horizontal edge, the interpolated curve and the lower horizontal edge have been used in a loft operation to construct a convex surface as shown in figure 5.

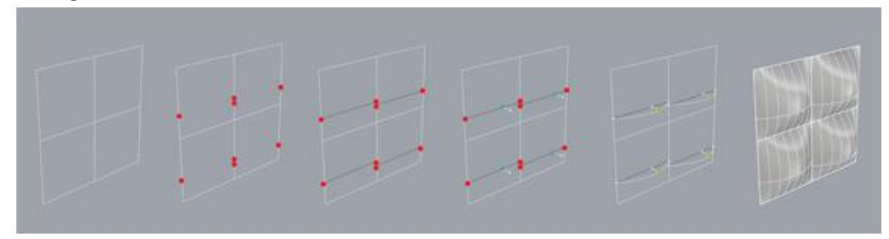

Fig. 5: Third design proposal.

\section{Results}

Each design proposal was submitted to simulation for blast load and pressure, the magnitude of the blast and the conditions of all cases were correspondent to those in the initial simulation except the form of the cube. Each simulation's results are compared with the initial simulation's results in order to reveal the differences in blast impact over each option, thus finding out whether or not the three parametric design proposals have mitigated the blast impact on the cube. The outcomes are as shown in figure 6 and table 1 .

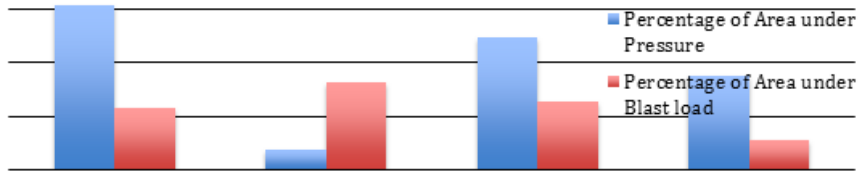

Fig. 6: Comparison of percentage of area between the initial simulation and the three proposals.

The results are two folds; first, in terms of pressure throughout the domain, it shows that, in general, pressure has decreased, hence, the number of affected surfaces is lower than the one in the initial simulation, though, the maximum pressure at some points has increased in comparison with the results of the initial simulation. This can be attributed to a number of factors listed below:
Table 1: Percentage Of Area Under Pressure And Blast Load

\begin{tabular}{|c|c|c|c|c|}
\hline$\%$ & $\begin{array}{c}\text { Simple } \\
\text { Cube }\end{array}$ & $\begin{array}{c}\text { Proposa } \\
101\end{array}$ & $\begin{array}{c}\text { Proposa } \\
102\end{array}$ & $\begin{array}{c}\text { Proposa } \\
103\end{array}$ \\
\hline $\begin{array}{c}\text { Percentage of Area } \\
\text { under Pressure }\end{array}$ & 30.85 & 3.73 & 24.77 & 17.49 \\
\hline $\begin{array}{c}\text { Percentage of Area } \\
\text { under Blast load }\end{array}$ & 11.56 & 16.3 & 12.76 & 5.56 \\
\hline
\end{tabular}

In the first proposal, although the number of affected subdivisions has decreased, a few subdivisions, specifically, around the corners of the cube have recorded higher pressure; this might be the result of reflection and accumulated overlapping waves.

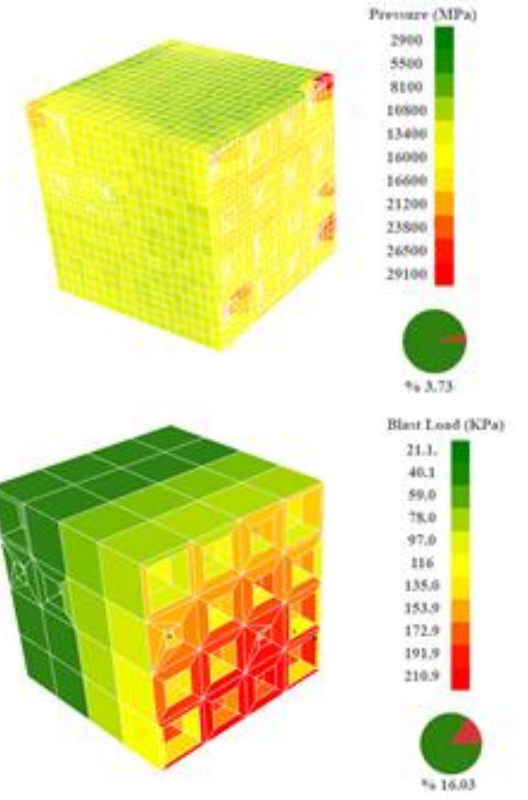

Fig. 7: First design proposal, pressure and blast load results

In the second proposal, similar to the first one, the total area of the affected surfaces has decreased, however, the maximum pressure on some surfaces has exceeded the one in the initial simulation. The surfaces with increased pressure as shown in figure 8 are usually those forming an angle of incidence $\left(40^{\circ}-\right.$ $60^{\circ}$ ) with the centre of explosion in addition to the form of the space that is left between each two pyramids-like subdivisions, these spaces are more likely to accumulate blast wave reflection and overlapped waves.

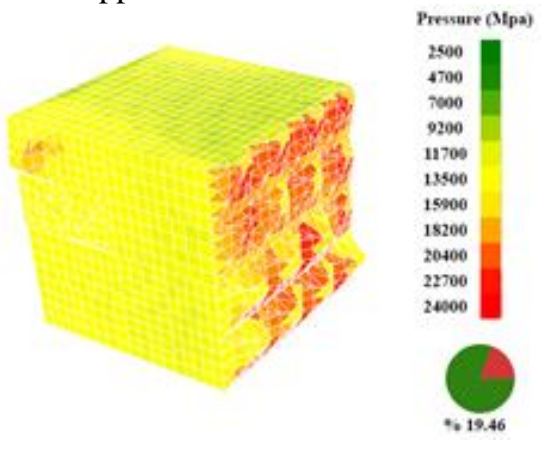




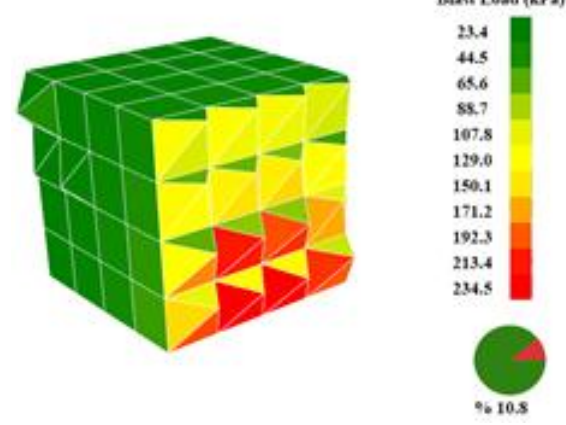

Fig. 8: Second design proposal, pressure and blast load results.

In the third proposal, the total area of affected surfaces has decreased while some surfaces have recorded a maximum pressure that is higher than the one in the initial simulation, in this case, similar to the previous ones, an accumulation of pressure has taken place in the joints in between the neighbouring subdivisions and also on those surfaces that form an angle of incident $40^{\circ}-60^{\circ}$ with the centre of the explosion.

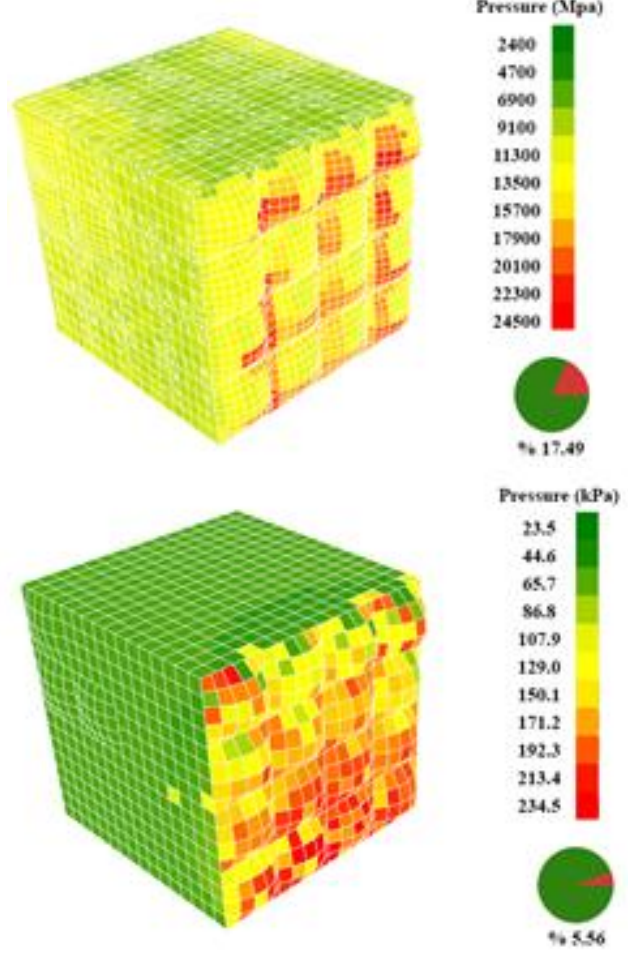

Fig. 9: Third design proposal, pressure and blast load results

In addition to the factors mentioned above, some parts of the subdivision, in option two and three, are closer to the centre of explosion in comparison to the cube in the initial experiment.

Second, regarding the blast load, the numerical analysis results show that the area of affected subdivision has increased in option one and two, while decreased in option three, however, the value of maximum blast load has decreased in option one, while increased in option two and three, the reason is that in option two and three the distance between the subdivisions with high blast load and the centre of explosion is shorter.

As a summary, apart of the role of shapes and forms in changing the blast impact, the peculiarity of parametric design in dealing with each part of the problem particularly while maintaining the interrelations between them, can further enhance the changes of blast impact on geometries regardless the change being positive or negative as this depends on the design concept and the extent to which this concept is informed by the principles of blast resistance design. Another advantage of parametric design is that even in the higher affected zones it is possible to create safer subzones as shown in figure 8 and 9 .

\section{CONCLUSIONS}

This paper has examined the capability of parametrically designed forms in reducing the impact of blast wave caused by bombs. This is a preliminary experiment towards broader applications on real buildings and urban elements. The aim is to make use of the recent advancements in computational design methods, specifically Parametric Design, in resisting such an intricate phenomenon that constitutes major risk for cities and buildings nowadays.

Three design proposals for a cubic form were experimented using the coyote tool; the results were, then, compared to the impact of the same blast (in terms of magnitude) on a simple flat faces cube with the same dimensions. The design concept of the three proposals was based on the existing literature on effective forms and shapes in resisting blast load and pressure. The results and analysis show that parametric design has the potential to reduce the total affected surface area, however some parts of the cube experienced higher pressure loads than the basic one, this implies further refinement in design in addition to further comprehension of the physical aspects and the nature of explosions.

\section{ACKNOWLEDGMENT}

The author expresses his greatest gratitude to ControlMad studio in Madrid for arranging a two days workshop about experimenting design proposals against blast impacts.

\section{REFERENCES}

[1] J. Yazdanseta \& M. Taheri, "Structural Design of Buildings to Resist Blast and Progressive Collapse (Case Study: Main Substation Building Located at Esfahan Refinery Plant in Iran)", WSEAS International Conference on Engineering Mechanics, Structures, Engineering Geology (EMESEG '08), Crete Island, Greece, 2008, p.271.

[2] S. B. Hosseini Bitarafian, M. hashemi-fesharaki and S. J. NorouzianMaleki, "The Role of Basic Forms Buildings in Explosion Protection", International Journal of Science and Advanced Technology, Vol. 2, No. 8, August 2012, pp. 47-50.

[3] N. Gebbeken and T. Döge, "Explosion Protection Architectural Design, Urban Planning and Landscape Planning", International Journal of Protective Structures, Vol. 1, Number 1, 2010, pp. 1-22. http://dx.doi.org/10.1260/2041-4196.1.1.1

[4] Federal Emergency Management Agency. FEMA 426: Reference Manual to Mitigate Potential Terrorist Attacks Against Buildings. FEMA, 2003, ch 4. pp. 1-20.

[5] A. D. Joseph, AIA, ed. Security, Planning and Design, A Guide for Architects and Building Design Professionals, John Wiley \& Sons, Inc., New Jersey, 2004.

[6] T. Norman, Understanding Threats in J.A. Demkin. Security Planning and Design. New Jersy: John wiley \& Sons, Inc. ${ }^{\text {st }}$ edition, 2004, pp. 21-35. 
[7] M.Y.H. Bangash \& T. Bangash, "Explosion-Resistant Buildings Design, Analysis, and Case Studies". London: Springer, $1^{\text {st }}$ edition, 2006.

[8] G.F. Kinney and K.J. Graham, Explosive Shocks in Air. Berlin: Springer, 1985. http://dx.doi.org/10.1007/978-3-642-86682-1

[9] G.C. Mays and P.D. Smith. Blast effects on buildings: Design of buildings to optimize resistance to blast loading. London: Thomas Telford, $2^{\text {nd }}$ edition, 1995.

[10] R. Woodbury, Elements of Parametric Design. New York: Routledge, 2010, pp. 11-12.

[11] P. Chias \& S. A. Noori, Digital Tools and Models of Computational Design Curiculum, presented at ARCHDESIGN'15, Istanbul, July 6-7, 2015. 